\title{
Immunohistochemical localization of aquaporin 4 (AQP4) in the porcine gastrointestinal tract
}

\author{
Marcin Bartłomiej Arciszewski ${ }^{1}$, Małgorzata Matysek ${ }^{1}$, Waldemar Sienkiewicz ${ }^{2}$ \\ ${ }^{1}$ University of Life Sciences, Faculty of Veterinary Medicine, Department of Animal Anatomy and Histology, \\ Lublin, Poland \\ ${ }^{2}$ University of Warmia and Mazury in Olsztyn, Faculty of Veterinary Medicine, \\ Department of Animal Anatomy, Olsztyn, Poland \\ Received October 11, 2014 \\ Accepted May 13, 2015
}

\begin{abstract}
The water channel aquaporin-4 (AQP4) is a protein widely expressed on plasma membrane of a variety of epithelial cells. In this study we investigated the expression of AQP4 in the gastrointestinal tract of the pig using immunohistochemical staining. We found no presence of AQP4 in the different regions of the pig stomach. In the porcine small intestine moderate immunoreactivity to AQP4 was detected in enterocytes (along the villi and in the bottom of the crypts), duodenal Brunner's glands and in enteric ganglia in cells lying in close vicinity to myenteric as well as submucous neurons. In superficial epithelial cells of the colonic mucosa as well as of caecal and colonic glands a very strong immunoreactivity to AQP4 was found. Both in the myenteric and submucous ganglia of the large intestine AQP4-positive cells surrounding enteric neurons were observed. We concluded that AQP4 expression in the porcine gastrointestinal tract showed some species-dependent differences in relation to other species. Based on the presented distribution pattern of AQP4, it is likely that the aquaporin plays a role in mucous (but not acid) secretion and intestinal absorptive processes in the pig.
\end{abstract}

Aquaporins, gut, small intestine, large intestine, pig

The term aquaporins refers to the group of major intrinsic proteins responsible for highly selective water transport across the cell membrane. The first aquaporin (AQP), formerly known as CHIP28, was discovered by Peter Agre's group in erythrocytes and proximal tubules of the rat kidney (Denker et al. 1988). But today the family of aquaporins has grown to at least 13 different ion channel membrane proteins (AQP0-AQP12) able to transport water as well as small solutes (King et al. 2004). The presence of some of aquaporins has been found to be limited to certain organs only, whereas others are widely expressed in numerous organs of several body systems (Takata et al. 2004). In recent years, at least several attempts have been made to determine the presence of aquaporins in the mammalian gastrointestinal tract (GIT), however, our knowledge about their distribution and role(s) is still far from satisfactory. Interestingly, it is evident that a number of aquaporin types can be found in the mammalian gut. Immunohistochemistry combined with RT-PCR studies indicated that mRNAs encoding AQP1, AQP3-5, AQP7-8 and AQP10-11 are commonly present in normal gastric tissues (Laforenza 2012). The use of specific primary antibodies raised against various types of aquaporins revealed the expression of AQP1, AQP4-5, AQP7, AQP9-11 in samples from the small intestine of certain animal species (Matsuzaki et al. 2004; Arciszewski et al. 2011; Jiang et al. 2014). In a healthy large intestine the presence of AQP3-4 and AQP8 transcripts has been confirmed with the use of RTPCR and Northern blot analysis (Ma and Verkman 1999; Elkjaer et al. 2001). It seems that such a specific distribution pattern of aquaporins makes them one of key players crucial for the physiological regulation of several processes including the most

Address for correspondence:

Prof. M.B. Arciszewski,

Department of Animal Anatomy and Histology

Faculty of Veterinary Medicine,University of Life Sciences

Akademicka 12, 20-033, Lublin, Poland

Phone: + 48 (81) 4456924

Fax: + 48 (81) 4456596

E-mail: mb.arciszewski@wp.pl

http://actavet.vfu.cz/ 
important one, the production and reabsorption of fluid (King et al. 2000), but also of dietary fat processing (Ma et al. 2001), gut inflammatory disorders (Zhao et al. 2014), tumour development (Papadopoulos and Saadoun 2014), or even autoimmune diseases (Pisani et al. 2014). Recent studies suggest that aquaporins may serve as an attractive novel drug target with a potential clinical application (Frigeri et al. 2007). A growing number of studies demonstrate that AQP4 has gained much more attention than the remaining family members, mainly due to its involvement in numerous pivotal life processes. AQP4 is predominantly expressed in the brain (Nielsen et al. 1997) and believed to be involved in the development of brain oedema and neuropsychiatric disorders (Wang et al. 2014; Xiao and $\mathrm{Hu}$ 2014). In a wider perspective, AQP4 is also a potent factor influencing the course of chronic inflammation of nasal mucosa (Frauenfelder et al. 2014), optic neuropathy (Petzold and Plant 2014), and secretion in diabetic prostate (Pei et al. 2013). Several evidences also linked AQP4 with gastrointestinal disorders. It must be, however, kept in mind, that most morphological and functional studies have been conducted in laboratory rodents and humans, whereas large animal species have attracted relatively lower attention. Considering the above mentioned facts, the present study was aimed to immunohistochemically investigate the occurrence, distribution and cellular localization of AQP4 in particular segments of the GIT of the pig, and thus to determine whether AQP4 might be a potential regulator of gastrointestinal disorders in this species.

\begin{abstract}
Animals and tissues
Materials and Methods

A total of five $(n=5)$ five-week-old crossbreed Polish Landrace Pietrain piglets (weighing approx. 4-5 kg) of both sexes, born at term and housed in standard farming conditions were used in the study. All experimental protocols described below were carried out in accordance with the Polish law regarding experimental animals, after having been reviewed and approved by the Local Ethics Committee. In order to minimize the unnecessary suffering of animals the number of animals used was reduced to the minimum needed to achieve meaningful results. On arrival the piglets were clinically healthy. Piglets were sedated with intramuscular injection of azaperone (Stresnil, Janssen-Cilag GmbH, Germany; $0.5 \mathrm{mg} / \mathrm{kg}$ b.w.) and euthanized by an overdose of sodium pentobarbital (Morbital, Biowet Pulawy, Poland; $50 \mathrm{mg} / \mathrm{kg}$ b.w.). A midline incision of the abdomen was made and samples of the stomach (divided into cardia, fundus, body, and pylorus), duodenum, jejunum, ileum, caecum, and colon samples (approx. $3 \mathrm{~cm}$ long) were collected from each animal immediately after killing. The tissue samples were immediately immersed in $10 \%$ phosphate buffered neutral formalin for $6 \mathrm{~h}$ at room temperature (RT) and then overnight at $4{ }^{\circ} \mathrm{C}$. Once, the fixative was washed the material was dehydrated through ascending grades of ethyl alcohol, cleared in xylene, and infiltrated with paraffin. Finally the material was embedded into paraffin blocks and cut using a microtome into $5 \mu \mathrm{m}$ thick sections. Sections were mounted on adhesion glass slides (SuperFrost Plus, Menzel GmbH \& CoKG, Germany). In order to completely remove water from the slides, each slide was left at RT overnight.
\end{abstract}

\title{
Immunohistochemistry
}

For the routine indirect immunohistochemical stainings peroxidase-antiperoxidase (PAP) method was applied. Slides were dewaxed in three xylene baths for $5 \mathrm{~min}$ each and hydrated in descending ethanol series for 5 min in each bath. After washing in distilled water the slides were placed in a plastic staining dish containing citrate buffer $(\mathrm{pH}=6.0)$. Antigen retrieval was performed at $97^{\circ} \mathrm{C}(3 \times 7 \mathrm{~min})$ in a microwave oven $(800 \mathrm{~W})$, followed by cooling for $20 \mathrm{~min}$. Next, the sections were treated with $3 \%$ hydrogen peroxidase for 20 min, rinsed in phosphate buffered saline (PBS, $\mathrm{pH}=7.4$ ) and incubated in $2.5 \%$ normal horse serum (S-2012; Vector, USA) for 20 min at RT. Incubation with mouse monoclonal antibodies (clone [3D2]) raised against AQP4 (1:200; Abcam, UK, code ab11026) was done overnight (humid chamber, $\left.4{ }^{\circ} \mathrm{C}\right)$. Next day, the sections were washed twice in PBS (15 min each) and then incubated for $1 \mathrm{~h}$ (RT) with anti-mouse/rabbit Ig (ImPress ${ }^{\mathrm{TM}}$; MP-7500 Vector, USA). Washing with PBS $(2 \times 15 \mathrm{~min})$ was repeated and immunoreaction was visualized by soaking samples in diaminobenzidine (DAB, Vector, USA) solution containing hydrogen peroxide. After the final rinse in tap water the sections were lightly counterstained with Mayer's haematoxylin solution, dehydrated in ascending series of ethyl alcohol, transferred to xylene and coverslipped.

In control stainings the primary antibodies were substituted by PBS or non-immune rabbit and mouse serum at an equal concentration as the primary antibodies. Omission of primary antibodies also served as a negative control. In control sections, no positive immunoreactions were found.

The sections were viewed under a light microscope (BX-51 DSU Olympus, Japan) equipped with a digital 
colour camera (DP-70, Olympus) and connected to a standard PC. Image acquisition and analysis were performed using $\mathrm{Cell}^{\wedge} \mathrm{M}$ software (Olympus).

\section{Results}

In general, as a result of the immunohistochemical staining DAB formed a deep brown polymerization product upon reaction with hydrogen peroxide in the presence of peroxidases, easily distinguishable in relation to the background. The intensity of DAB staining was diverse in particular organs of the porcine GIT. In all histological layers (muscularis externa, submucosa and mucosa) of all studied regions of the porcine stomach (cardia, fundus, body and pylorus) we were unable to detect positive immunoreactivity to AQP4. In gastric enteric ganglia neither AQP4-immunoreactive (IR) neurons nor AQP4IR dots/varicosities running in close vicinity to neurons were found. In the duodenum, the expression of AQP4 was found in numerous enterocytes located all along the villi and in the bottom of the duodenal crypts (Plate I, Fig. 1A). Clusters of dots intensively immunoreactive to AQP4 were also detected in cells of Brunner's glands (Plate I, Fig $1 \mathrm{~B})$ as well as in very sparse cells surrounding enteric neurons. In the jejunum and ileum numerous AQP4-immunoreactive enterocytes were found in the upper portion of the villi. Additionally, cells located in the basal part of crypts, with visibly weaker immunoreaction to AQP4 were also occasionally noted. Expression of AQP4 along the upper part of the jejunal villi was frequently noted (Plate I, Fig 1C). In the myenteric and submucous ganglia (predominantly of the outer submucous plexus), numerous AQP4-IR cells (most likely glial cells) lying in close vicinity to enteric neurons were detected (Plate I, Fig 1D). No presence of AQP4-positive structures was found in the ileal Payer's patches. In the caecum, immunoreactivity to AQP4 was found in apical part of crypt epithelial cells only (Plate II, Fig 2A). A very strong immunoreaction to AQP4 was present in both basolateral and apical regions of the colonic epithelial cells (Plate II, Fig. 2B). Similar distribution pattern of AQP4-expression was also noted in case of colonic intestinal glands (Plate II, Fig. 2C). In myenteric and submucous ganglia of the colon only sparse AQP4-IR cells with morphology resembling glial cells were found around enteric neurons.

\section{Discussion}

In the present paper, we for the first time described the detailed expression pattern to AQP4 in the porcine gastrointestinal tract (GIT). Aquaporin 4 (AQP4) belongs to the subgroup of orthodox aquaporins responsible for transcellular transport of water only (Borgnia et al. 1999). AQP4 is widely present in the brain, predominantly in astrocytes (Nagelhus and Ottersen 2013), however, recent findings documented AQP4-expression in the mammalian GIT. Thus, the most obvious and reasonable roles of AQP4 in GIT seem to be secretion and absorption which principally depend on the in and out transmembrane water flux. Based on several findings, we can conclude that the presence of AQP4 in the gut of the pig seems to be speciesspecific and thus its physiological role might be slightly different. First of all, in the present study we were unable to localize AQP4 in any of the cells of different regions of the porcine stomach (cardia, fundus, body, and pylorus). In previous studies, the expression of AQP4 was detected at basolateral membrane of parietal cells located in the stomach of the rat (Fujita et al. 1999), mouse (Wang et al. 2000), guinea pig (Jiang et al. 2014) and humans (Misaka et al. 1996). Since the application of proton pump inhibitors evoking acid suppression substantially increased the number of fundic AQP4-expressing parietal cells in the mouse, the involvement of aquaporin in gastric secretory processes (acid secretion) has been postulated (Matsuzaki et al. 2010). This is in line with previous findings noting a higher expression level of AQP4 in the stomach (due to increased number of AQP4-positive parietal cells) of peptide YY transgenic mice (Carmosino et al. 2005). 
In the present study we also reported the immunoreactivity to AQP4 in the distinct parts of the porcine small intestine. The most intensive AQP4-expression was noted in the epithelial margin of the small intestine (duodenum, jejunum, and ileum), however, the presence of AQP4 was also visible in crypts and duodenal Brunner's glands. The presented herein AQP4 distribution pattern with a small exception closely resembles those previously described in other mammals. In the rat duodenum, jejunum and ileum the immunoreactivity to AQP4 was found at basolateral membranes of the crypt cells but not along the villi (Koyama et al. 1999). On the other hand, by means of immunofluorescence and Western blot techniques, weak or moderate expressions of AQP4 were found both in the guinea-pig small intestinal crypts and villi (Jiang et al. 2014). The cellular distribution pattern of AQP4 in the porcine small intestine suggests its involvement in absorptive processes. Additionally, since a weak expression of AQP4 was also found in porcine Brunner's glands it is also likely that AQP4 participates in mucus production. Mechanisms underlying these processes are not fully understood, but it has been previously shown that AQP4-regulated water flux in the small intestine of humans increases after cholera toxin or forskolin stimulation, which suggests that AQP4 controls water movements by increase of intracellular cAMP concentration (Hamabata et al. 2002). It has been also well documented that there is a functional correlation between the intestinal dysfunction (oedema) and traumatic brain injury, which is believed to be regulated by the increased level of AQP4 in the small intestinal mucosa (Duan et al. 2013).

The expression pattern of AQP4 in the large intestine of several species has been well documented. Strong AQP4 immunostaining in membranes of the epithelial cells at luminal surface was reported in the colon of the mouse (Wang et al. 2000) and guinea-pig (Jiang et al. 2014), which is in line with the results obtained herein from the pig caecum and colon. The presence of AQP4 protein in mouse colon was additionally confirmed with the use of Western blot analysis (Cao et al. 2014). It must be, however, kept in mind that in the rat colon no immunoreaction for AQP4 was detected (Koyama et al. 1999) and human colon contains only low levels of AQP4 mRNA (Laforenza 2012), which strongly points to the existence of species-dependent differences. Since transepithelial osmotic water permeability in the colon of mice lacking AQP4 gene was significantly decreased, it is generally accepted that AQP4 plays a role in the regulation of the colonic fluid flow (Wang et al. 2000). In mice infected with the rotavirus strain SA11 (animal model of diarrhoea) as well as ovalbumin-sensitized mice (animal model of food allergy) the protein content of AQP4 was significantly attenuated in the colon (Yamamoto et al. 2007; Cao et al. 2014). In humans, lower expression of AQP4 in colonic mucosa is correlated with the development of slow transit constipations (Wang et al. 2010). Changes in expression levels of AQP4 were additionally observed in inflammatory diseases such as colitis (Hansen et al. 2009), ulcerative colitis and Crohn's disease (Hardin et al. 2004).

In several previous studies, the expression of AQP4 in the enteric nervous system (ENS) was presented, however, the results are sometimes contradictory. In rat and mouse colon populations of AQP4-immunoreactive myenteric as well as submucous neurons additionally co-expressing neurofilament $\mathrm{H}$ (neuronal marker) were found (Thi et al. 2008). On the other hand, in the ENS of the guinea-pig the presence of AQP4-positive glial cells (co-expressing a glial marker S100ß) with simultaneous absence of AQP4positive enteric neurons was reported (Jiang et al. 2014). Interestingly, another study on human GIT samples revealed that AQP4-immunoreactive structures present in the enteric plexuses are negative for glial fibrillary acidic protein, which allows the authors to classify them as enteric neurons (Gao et al. 2006). In the present study we found no AQP4 immunoreactivity in either myenteric or submucous neurons of the stomach, small or large intestines, however, in the studied enteric ganglia the presence of AQP4-positive cells in close neighbourhood to neurons was noted. Whether the observed AQP4-IR cells 
are in fact enteric glial cells is a matter of speculation at the moment and needs more intensive morphological studies. It must be kept in mind that from the structural point of view, enteric glia are very similar to astrocytes in the central nervous system (Jessen and Mirsky 1983) and AQP4 is expressed in foot processes of astrocytes throughout the central nervous system (Tomassoni et al. 2010).

In conclusion, our data demonstrate that AQP4 expression in the gut of the pig is species-related. The peculiar distribution pattern of AQP4 in the porcine GIT suggests that aquaporins are important regulators of proper gut functioning in this animal species. Further functional studies involving AQP4-selective pharmacological agents may improve our understanding of pathogenesis of the porcine intestinal disorders.

\section{References}

Arciszewski MB, Stefaniak M, Zacharko-Siembida A, Całka J 2011: Aquaporin 1 water channel is expressed on submucosal but not myenteric neurons from the ovine duodenum. Ann Anat 193: 81-85

Borgnia M, Nielsen S, Engel A, Agre P 1999: Cellular and molecular biology of the aquaporin water channels. Annu Rev Biochem 68: 425-458

Cao M, Yang M, Ou Z, Li D, Geng L, Chen P, Chen H, Gong S 2014: Involvement of aquaporins in a mouse model of rotavirus diarrhea. Virol Sin 29: 211-217

Carmosino M, Mazzone A, Laforenza U, Gastaldi G, Svelto M, Valenti G 2005: Altered expression of aquaporin 4 and $\mathrm{H}(+) / \mathrm{K}(+)$-ATPase in the stomachs of peptide YY (PYY) transgenic mice. Biol Cell 97: 735-742

Denker BM, Smith BL, Kuhajda FP, Agre P 1988: Identification, purification, and partial characterization of a novel Mr 28,000 integral membrane protein from erythrocytes and renal tubules. J Biol Chem 263: 15634-15642

Duan H, Hao C, Fan Y, Wang H, Liu Y, Hao J, Xu C, Liu X, Zhang H 2013: The role of neuropeptide Y and aquaporin 4 in the pathogenesis of intestinal dysfunction caused by traumatic brain injury. J Surg Res 184: 1006-1012

Elkjaer ML, Nejsum LN, Gresz V, Kwon TH, Jensen UB, Frøkiaer J, Nielsen S 2001: Immunolocalization of aquaporin-8 in rat kidney, gastrointestinal tract, testis, and airways. Am J Physiol Renal Physiol 281: F1047-F1057

Frauenfelder C, Woods C, Hussey D, Ooi E, Klebe S, Carney AS 2014: Aquaporin expression profiles in normal sinonasal mucosa and chronic rhinosinusitis. Int Forum Allergy Rhinol doi: 10.1002/alr.21415

Frigeri A, Nicchia GP, Svelto M 2007: Aquaporins as targets for drug discovery. Curr Pharm Des 13: 2421-2427

Fujita A, Horio Y, Nielsen S, Nagelhus EA, Hata F, Ottersen OP, Kurachi Y 1999: High-resolution immunogold cytochemistry indicates that AQP4 is concentrated along the basal membrane of parietal cell in rat stomach. FEBS Lett 459: 305-309

Gao H, He C, Fang X, Hou X, Feng X, Yang H, Zhao X, Ma T 2006: Localization of aquaporin-1 water channel in glial cells of the human peripheral nervous system. Glia 53: 783-787

Hamabata T, Liu C, Takeda Y 2002: Positive and negative regulation of water channel aquaporins in human small intestine by cholera toxin. Microb Pathog 32: 273-277

Hansen JJ, Holt L, Sartor RB 2009: Gene expression patterns in experimental colitis in IL-10-deficient mice. Inflamm Bowel Dis 15: 890-899

Hardin JA, Wallace LE, Wong JF, O’Loughlin EV, Urbanski SJ, Gall DG, MacNaughton WK, Beck PL 2004: Aquaporin expression is downregulated in a murine model of colitis and in patients with ulcerative colitis, Crohn's disease and infectious colitis. Cell Tissue Res 318: 313-323

Jessen KR, Mirsky R 1983: Astrocyte-like glia in the peripheral nervous system: an immunohistochemical study of enteric glia. J Neurosci 3: 2206-2218

Jiang L, Li J, Liu X, Burnstock G, Xiang Z 2014: Expression of aquaporin-4 water channels in the digestive tract of the guinea pig. J Mol Histol 45: 229-241

King LS, Yasui M, Agre P 2000: Aquaporins in health and disease. Mol Med Today 6: 60-65

King LS, Kozono D, Agre P 2004: From structure to disease: the evolving tale of aquaporin biology. Nat Rev Mol Cell Biol 5: 687-698

Koyama Y, Yamamoto T, Tani T, Nihei K, Kondo D, Funaki H, Yaoita E, Kawasaki K, Sato N, Hatakeyama K, Kihara I 1999: Expression and localization of aquaporins in rat gastrointestinal tract. Am J Physiol 276: C621-C627

Laforenza U 2012: Water channel proteins in the gastrointestinal tract. Mol Aspects Med 33: 642-650

Ma T, Verkman AS 1999: Aquaporin water channels in gastrointestinal physiology. J Physiol 517: 317-326

Ma T, Jayaraman S, Wang KS, Song Y, Yang B, Li J, Bastidas JA, Verkman AS 2001: Defective dietary fat processing in transgenic mice lacking aquaporin-1 water channels. Am J Physiol Cell Physiol 280: C126-C134

Matsuzaki J, Suzuki H, Minegishi Y, Sugai E, Tsugawa H, Yasui M, Hibi T 2010: Acid suppression by proton pump inhibitors enhances aquaporin-4 and KCNQ1 expression in gastric fundic parietal cells in mouse. Dig Dis Sci 55: $3339-3348$ 
Matsuzaki T, Tajika Y, Ablimit A, Aoki T, Hagiwara H, Takata K 2004: Aquaporins in the digestive system. Med Electron Microsc 37: 71-80

Misaka T, Abe K, Iwabuchi K, Kusakabe Y, Ichinose M, Miki K, Emori Y, Arai S 1996: A water channel closely related to rat brain aquaporin 4 is expressed in acid- and pepsinogen-secretory cells of human stomach. FEBS Lett 381: 208-212

Nagelhus EA, Ottersen OP 2013: Physiological roles of aquaporin-4 in brain. Physiol Rev 93: 1543-1462

Nielsen S, Nagelhus EA, Amiry-Moghaddam M, Bourque C, Agre P, Ottersen OP 1997: Specialized membrane domains for water transport in glial cells: high-resolution immunogold cytochemistry of aquaporin-4 in rat brain. J Neurosci 17: 171-180

Papadopoulos MC, Saadoun S 2014: Key roles of aquaporins in tumor biology. Biochim Biophys Acta doi: 10.1016/j.bbamem.2014.09.001

Pei L, Yang G, Jiang J, Jiang R, Deng Q, Chen B, Gan X 2013: Expression of aquaporins in prostate and seminal vesicles of diabetic rats. J Sex Med 10: 2975-2985

Petzold A, Plant GT 2014: Diagnosis and classification of autoimmune optic neuropathy. Autoimmun Rev 13: 539-545

Pisani F, Mola MG, Simone L, Rosito S, Alberga D, Mangiatordi GF, Lattanzi G, Nicolotti O, Frigeri A, Svelto M, Nicchia GP 2014: Identification of a point mutation impairing the binding between aquaporin-4 and the neuromyelitis optica autoantibodies. J Biol Chem doi: 10.1074/jbc.M114.582221

Takata K, Matsuzaki T, Tajika Y 2004: Aquaporins: water channel proteins of the cell membrane. Prog Histochem Cytochem 39: 1-83

Thi MM, Spray DC, Hanani M 2008: Aquaporin-4 water channels in enteric neurons. J Neurosci Res 86: 448-456

Tomassoni D, Bramanti V, Amenta F 2010: Expression of aquaporins 1 and 4 in the brain of spontaneously hypertensive rats. Brain Res 1325: 155-163

Wang G, Yuan Y, Zhang J, Gao L, Tan X, Yang G, Lv X, Jin Y 2014: Roles of aquaporins and matrix metalloproteinases in mouse brain edema formation induced by subacute exposure to 1,2-dichloroethane. Neurotoxicol Teratol 44: 105-112

Wang KS, Komar AR, Ma T, Filiz F, McLeroy J, Hoda K, Verkman AS, Bastidas JA 2000: Gastric acid secretion in aquaporin-4 knockout mice. Am J Physiol Gastrointest Liver Physiol 279: G448-G453

Wang XJ, Yuan WT, Song JM, Zhang ZY 2010: Expression and significance of aquaporin 4 in the colonic mucosa of patients with slow transit constipation. Zhonghua Wei Chang Wai Ke Za Zhi 13: 445-447

Xiao M, Hu G 2014: Involvement of aquaporin 4 in astrocyte function and neuropsychiatric disorders. CNS Neurosci Ther 20: 385-390

Yamamoto T, Kuramoto H, Kadowaki M 2007: Downregulation in aquaporin 4 and aquaporin 8 expression of the colon associated with the induction of allergic diarrhea in a mouse model of food allergy. Life Sci 81: 115-120

Zhao G, Li J, Wang J, Shen X, Sun J 2014: Aquaporin 3 and 8 are down-regulated in TNBS-induced rat colitis. Biochem Biophys Res Commun 443: 161-166 
Plate I

Arciszewski M. B. et al.: Immunohistochemical ... pp. 321-326
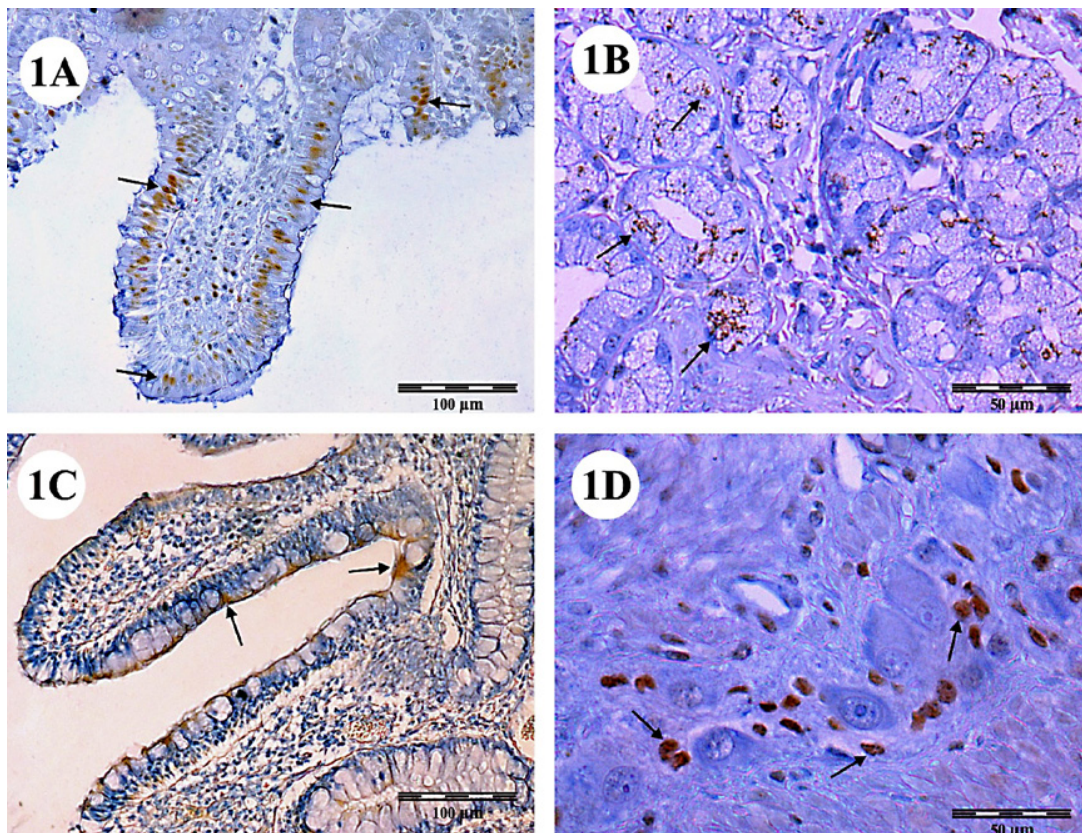

Fig. 1. Representative images of staining for AQP4 in the small intestine of the pig: (A) AQP4-expressing enterocytes found in duodenum are marked with arrows, (B) AQP4-containing structures located in duodenal Brunner's glands are marked with arrows, $(C)$ in upper portion of jejunal villi immunoreactivity to AQP4 is marked with arrows, (D) arrows point to AQP4-positive cells lying in close vicinity to submucous neurons. 

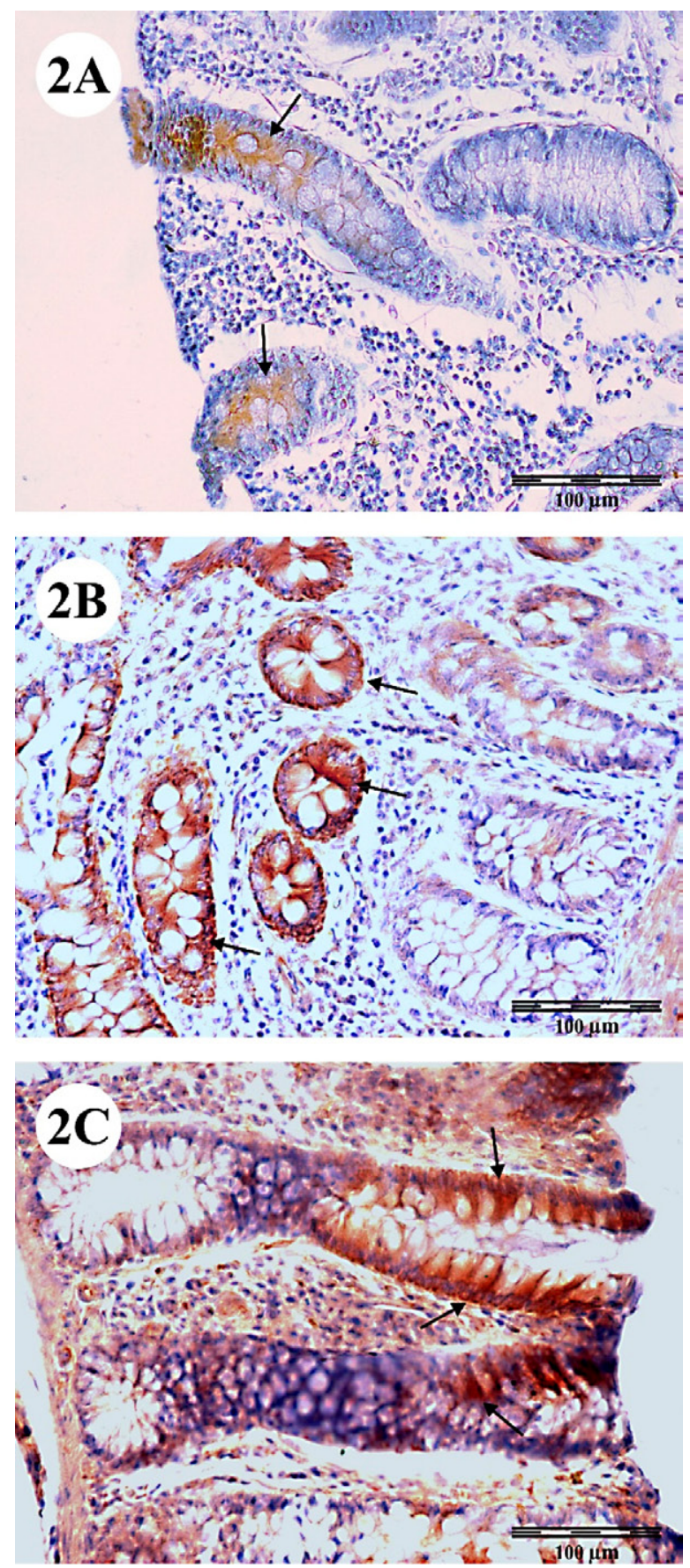

Fig. 2. Sections of the porcine large intestine immunostained for AQP4. In the caecum (A) expression to AQP4 (arrows) was observed in the upper part of crypt epithelial cells. In both colonic intestinal glands (arrows in B) as well as colonic epithelial cells (arrows in C) strong immunoreactivity to AQP4 was found. 